\title{
A Lightweight Docking Station for a Hovering AUV
}

\author{
Nuno A. Cruz, Aníbal C. Matos, Rui M. Almeida and Bruno M. Ferreira \\ Faculty of Engineering, University of Porto and INESC TEC \\ nacruz@fe.up.pt, anibal@fe.up.pt, rui.almeida@fe.up.pt, bm.ferreira@fe.up.pt
}

\begin{abstract}
The concept of underwater docking stations has long been proposed to support the long term deployment of AUVs, but the number of successful solutions is still very disappointing. Hovering type AUVs can navigate arbitrarily slow, simplifying the docking maneuver and the requirements for the receiving structure. This paper describes a docking system that was developed to extend the mission duration of the MARES AUV, a man portable hovering type AUV. Given the wide range of operational scenarios and configurations of this AUV, one of the design requirements was to have a simple modular structure, that could easily be reconfigured to support different vehicle configurations, deployment scenarios and docking maneuvers. The paper provides details of the mechanical aspects, the onboard electronic subsystems, and the general operational procedure, as well as preliminary data from the first trials.
\end{abstract}

\section{INTRODUCTION}

The concept of underwater docking stations has been suggested with the improvement of the operational level of AUVs, anticipating scenarios in which these autonomous vehicles could operate unattended for very long periods of time. Such underwater garages were idealized to charge batteries, to transfer information at high data rates, and also to provide shelter when sea state increased.

Since most early AUVs were "flying type" vehicles, with horizontal propulsion and control fins, they had a minimum forward velocity to ensure controllability. This complicated dramatically the docking maneuver, as the vehicle had to home in some sort of docking cone or structure, demanding very high accuracy with a short time to process any local navigation data, often resulting in strong impacts with the receiving structure. Furthermore, all power and data transfers were also relatively complex with such configurations and this partly explains the relatively disappointing number of successful operational docking stations for flying type AUVs.

Hovering type AUVs can navigate at arbitrarily low velocities, and most can even reverse velocity. This means that during a docking maneuver all data processing can be more complex, the vehicles can approach the docking structure only when the level of confidence is high, and they have many chances to retry the maneuver if needed. On the other hand, the vehicle configuration allows for other types of docking structure apart from the "docking cone", for example sideways or in the vertical direction.

This paper describes a docking system that was developed for the MARES AUV, a man portable hovering type AUV [1]. The main objective of the docking station is, naturally, to extend vehicle missions by providing means to charge the batteries and also to exchange large data files.

The paper is organized as follows. Section II provides an overview of the main efforts that have been made to develop operational concepts of underwater docking stations for AUVs. Section III summarizes explicitly the main requirements for the development of this new docking station. Section IV details the components of the docking station, both in terms of mechanical arrangement and also in terms of electronic subsystems. Section VI describes the AUV components that are required to ensure a proper docking maneuver and an example of a possible docking algorithm or procedure using the system components. Finally, in Section VII we present the first results from our experimental validation, and lastly, in Section VIII we present some concluding remarks and future research directions.

\section{RELATED WORK}

Docking stations are generally seen as underwater garages for AUVs to recharge their batteries and exchange data, and they are fundamental for extended deployments at sea. They can be divided in four main types, depending on deployment configuration: Surface docks, bottom-mounted docks, hanging docks and towed docks. The type of dock is usually dictated by the operational scenario, therefore some multipurpose vehicles may require hybrid docks which can operate in different configurations. The design of a docking system is greatly influenced by the type of AUV, paying due attention to issues like robustness and stability. The first prototypes of underwater docking stations were designed for flying type AUVs, that would home in to a cone or pole, relying on ultra short baseline acoustics for the final approximation ( [2], [3]). This approach is still being perfected for flying type AUVs, with very good results in various operational conditions [4], [5].

In the last years, there has been a proliferation of hovering type AUVs, yielding a substantial increase in maneuverability. At the same time, current onboard processing capabilities permit real time analysis of complex data, like video images. This is fundamental to provide accurate data to feed a close range controller for the final docking sequence. In fact, and due to sensing constraints, the same technological solutions cannot be adopted for both long- and short-range relative localization. Long-range relative localization is typically achieved by means of ranging, bearing or a combination of both (see, for example, [6]-[8]). Using this sensing technology makes it possible 
to achieve errors in the order of some tens of centimeters, typically depending on the distance itself. This is enough for relatively long range navigation, with effective ranges up to a few kilometers. However, in short-range (less than a few meters), this localization approach becomes ineffective because the error is too large for precise positioning. Complementarily, vision-based localization becomes more effective in short-range, enabling accurate relative localization. Previous works have validated this approach [9], [10], characterizing the error using a visual marker and closing the loop by precisely positioning an AUV relative to a marker.

This complementary navigation scheme has been the preferred choice for most recent docking systems for hovering AUVs [11]-[14]. In [12], it is assumed that the AUV is capable of performing smooth and very controlled movements. The docking procedure is accomplished by roughly approaching the dock via acoustic guidance and switching to visual mode to complete the maneuver using two sets of visual markers. For power transfer, an underwater-pluggable connector is used. The connector is mounted between two guidance bolts. A linear spindle engine is used to move the mechanism and couple plug and socket. Two modified COTS WiFi routers are used for data transfer. Tests conducted in a water tank resulted in bandwidth of up to $30 \mathrm{Mb} / \mathrm{s}$ across a distance of $8 \mathrm{~cm}$. Another docking system for hovering type AUVs was presented in [13]. The dock is a bottom-mounted open frame assembly equipped with an acoustic transducer and a led marker. The docking method requires an AUV fitted with acoustic positioning and a forward looking camera. The vehicle approaches the dock by acoustic localization and at a given distance it switches to visual positioning. The docking maneuver is accomplished relatively to the LED marker, positioned in the dock's frame. In [14], an underwater observatory with a docking system for long term environmental observation was described. The docking system is a sea-floor mounted version and is equipped with guiding LEDs, a magnetic modem for communications and a wireless charging system. The AUV uses stereo cameras to detect the docks LEDs and perform the docking maneuver. With the vehicle in place up to $1 \mathrm{~kW}$ of power is transferred through the wireless system and data transfer is assured by the magnetic modems.

\section{SYSTEM REQUIREMENTS}

The MARES AUV is a hovering type vehicle assembled with $20 \mathrm{~cm}$ diameter modular sections, resulting in lengths between $1.5 \mathrm{~m}$ and $2 \mathrm{~m}$, and weights between $30 \mathrm{~kg}$ and $40 \mathrm{~kg}$. Part of the success of the MARES AUV is the portability and modularity of the system, which have enabled the repeated operation in a wide range of scenarios. For the development of the docking station, the main purpose was to come up with a platform to test different docking approaches for this AUV. This allowed us to determine the main requirements for the subsystems.

\section{A. Main Operational Requirements}

Given the wide range of operational scenarios and configurations of the MARES AUV, probably the main design requirement was to have a simple modular structure that could easily be reconfigured to support different vehicle configurations, deployment scenarios and docking maneuvers. This had to be ensured with a low-logistics system, to match that of the AUV and allow a simple deployment of the overall system. Ideally, the docking station should be deployed and recovered using a simple RHIB, which imposed considerable limitations on size, weight, and general handling means.

We have translated these general guidelines into specific constraints:

- lifting points for launch and recovery;

- total weight in air less than $40 \mathrm{~kg}$;

- maximum dimensions of $2 \mathrm{~m} \times 1 \mathrm{~m} \times 1 \mathrm{~m}$.

The envisaged operational scenarios were mainly for coastal waters, up to a few tens of meters of depth, with deployments lasting for a few days up to a few weeks (this exempted us from dealing with longer term issues like biofouling). Due to the variability of sea bottom types that may be encountered, another operational requirement was to have real time access to the attitude of the docking station, as well as a down-looking camera to perform a preliminary survey of the deployment location, right before landing (eventually, a complimentary altimeter could be installed to yield a direct distance to bottom). This had to be complemented to a real time status of the internal system, like battery level and internal temperature.

Regarding external connections, the docking station should have an Ethernet communication link, as well as an external power input to provide energy to the onboard electronics and charge the backup batteries. Both connections should allow the installation onto existing local observatories, taking advantage of available infrastructures, or, alternatively, to a surface buoy providing power and a gateway to shore.

In summary, these requirements led to another set of constraints for our design:

- downward looking video camera and lights;

- possibility of installing an altimeter;

- cabled connection to shore/surface: communications and power cable with a minimum of $30 \mathrm{~m}$;

- backup battery for at least 2 hours of operation;

- depth and attitude sensors;

- internal health system (leak, battery level, temperature);

- minimum 10 bar pressure hulls.

\section{B. Docking Maneuvers}

As described earlier, our modular structure should be able to support multiple docking strategies, and the possibility of placing the dock on the sea bed, floating above sea bed on hanging from the surface. For the AUV to be able to dock in a given operational scenario, first it needs to be able to find the dock. In fact, given the uncertainty associated with any underwater positioning system, it is virtually impossible 


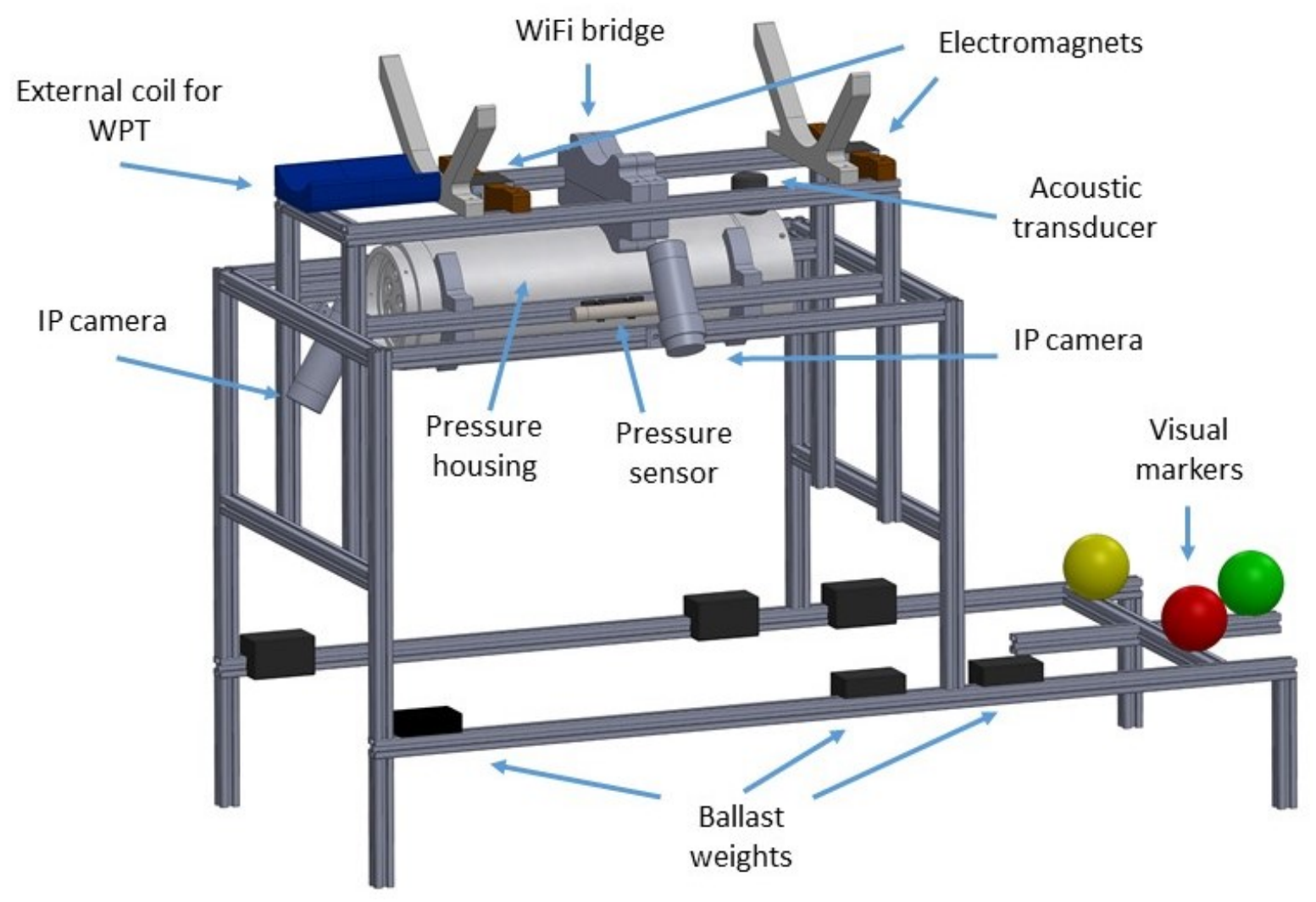

Fig. 1. Overview of the mechanical arrangement of the docking station.

to provide absolute coordinates of a [small] docking system, and expect an AUV to get to that exact position after traveling a few kilometers, for a few hours. For these reasons, we've decided to use a common approach, based on a complementary system with long range acoustic signals to approximate the docking station, and a close range visual system to guide the vehicle during the final stage of the docking maneuver. In order to document docking and undocking maneuvers, a video camera should also be installed on the docking structure. Video could be recorded locally and/or transmitted in real time to the surface.

In summary, the docking station should have some specific equipment to support the docking maneuvers:

- acoustic system for long range approach;

- visual target for short range positioning and guidance;

- lights to illuminate visual target;

- mechanical guidance and a locking mechanism to hold the AUV in place;

- upward looking video camera to document AUV operations and general state

\section{Requirements for Coupling}

For practical reasons and robustness, all power transfer and communications between the docking station and the AUV should be wireless. Moreover, the AUV should spend a minimum amount of energy while sitting at the dock, with the required energy being provided by the docking station. At the same time, the AUV should be able to communicate wirelessly while at the dock, and, at the same time, receive energy to charge the on board batteries.

In summary, the docking system and the AUV should have a distributed system with the following requirements:

- locking mechanism to overcome the net buoyancy of the AUV, with a minimum force of $10 \mathrm{~N}$;

- short range wireless communications between the docking station and the AUV;

- wireless power transfer from the docking station to the AUV.

\section{Docking Station Subsystems}

For the development of the docking station, we have fulfilled the above requirements with a mix of off-the-shelf parts and the reuse of specific components from other projects. This ensured a quick access to a working prototype that enabled the parallel development and validation of different algorithms and subsystems.

\section{A. Mechanical Structure}

The overall mechanical structure is based on modular aluminum profiles, an easily reconfigurable system with many solutions to attach sensors and actuators. A schematic representation can be seen in figure 1 . The overall envelope of this 


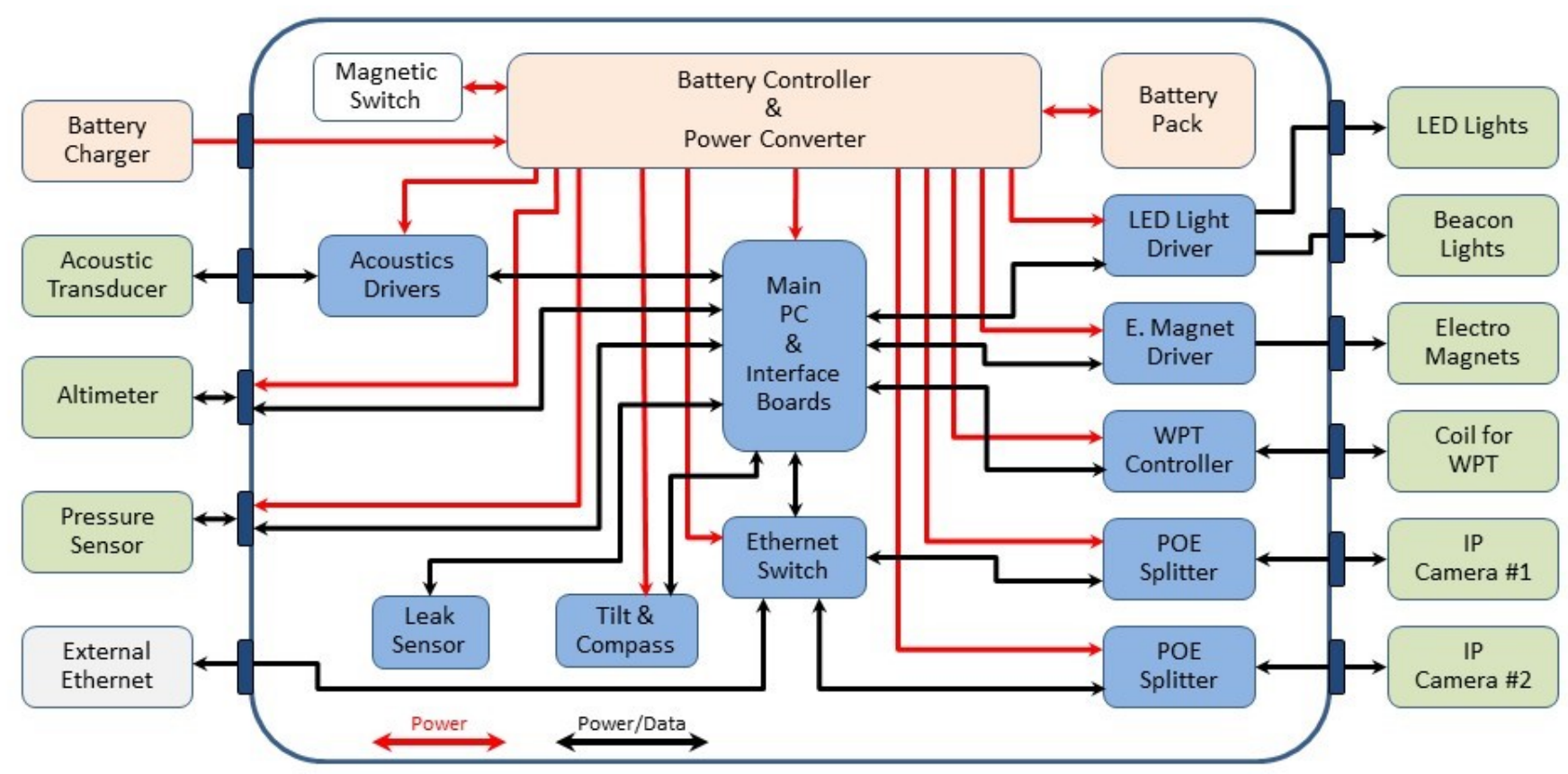

Fig. 2. Overview of the electronic subsystems and interconnections of the docking station.

configuration is $1.5 \mathrm{~m}$ long, $0.8 \mathrm{~m}$ wide and $1 \mathrm{~m}$ high, with a total weight close to $40 \mathrm{~kg}$. The initial structure was designed to land on the sea floor, with a low center of gravity adjusted with ballast weights, although it can later be lowered from the surface, if necessary. At the top layer, a docking cradle was installed to hold the vehicle with the aid of two electromagnets. A visual target with 3 colored globes (red, yellow, green) is attached to the bottom of this structure, with sufficient offset as to minimize visual occlusion during the docking maneuver. The geometry and location of the visual markers can be easily adjusted, but our most common configuration is a triangular shape with $40 \mathrm{~cm}$ of separation. An external LED light source was also installed to illuminate the target if required. All locations and offsets can be easily changed in a few minutes, by adjusting only a few set screws.

A pressure vessel is located underneath the AUV cradle, holding all electronic boards and energy in an internal aluminum frame. This pressure housing has the same design as the housings for our range of modular AUVs, as described in [15]. It is a $50 \mathrm{~cm}$ long, $20 \mathrm{~cm}$ diameter, polyacetal cylinder that withstands 20 bar of hydrostatic pressure. Using the same design as the AUVs' pressure housings not only accelerates development, but also facilitates the installation and test of sensors for long periods of time prior to their integration on AUVs.

\section{B. On Board Electronics}

An overview of the electronic subsystems and interconnections of the docking station can be seen in figure 2 .

Inside the pressure housing, a simple single board computer (SBC) manages all subsystems, with the aid of a few OEM interface boards. The current configuration is based on a
Raspberry Pi running Linux, with a solid state disk to log all activities. These include time stamped data from sensors, as well as a registry of actuation commands. The interface boards convert two of the USB ports of the SBC into RS232, RS485, and TTL serial, as required by the different subsystems. Although the pressure housing is connected to the surface, it has its own Li-Po rechargeable batteries to ensure continuous autonomous operation. They provide a total of $110 \mathrm{Wh}$ at a nominal voltage of $13.2 \mathrm{~V}$, and this voltage is converted down to $3.3 \mathrm{~V}, 5 \mathrm{~V}$, and $12 \mathrm{~V}$, as required by the electronic subsystems. A simple internal supervision system monitors battery level, as well as other safety parameters like internal temperature and water presence.

Specific to the docking maneuver are the devices used to facilitate the identification of localization and pose of the docking station by the AUV, including a set of visual markers and an acoustic system. The visual markers are illuminated with internal LEDs that can be dimmed as required to adapt to the local visibility conditions. The acoustic system has a receiver capable of decoding incoming frequencies, in the range of $20-30 \mathrm{kHz}$, and a transmitter in the same range. Together, they can be used in different ways to support multiple localization approaches. If the system is configured as a transponder, it will reply to a specific signal with another signal, allowing the "interrogator" to measure time of flight and therefore to estimate distance. This can serve to determine the location of the docking system upon deployment, by ranging from multiple known positions. During the AUV approach to docking, this can also work for the AUV to home to the target, using techniques called "single beacon navigation". 
At the final stage of the docking maneuver, the AUV slowly descends towards the cradle, and the electromagnets are activated and hold the vehicle in position. The Wireless Power Transfer (WPT) system can then be powered, to transfer energy to the AUV. Additionally, the pressure housing also includes a WiFi access point that allows close range communication with the AUV when docked, to allow for AUV data download and new mission upload.

All these electronic boards are installed inside the pressure housing, and there is still available volume for additional subsystems (fig. 3).

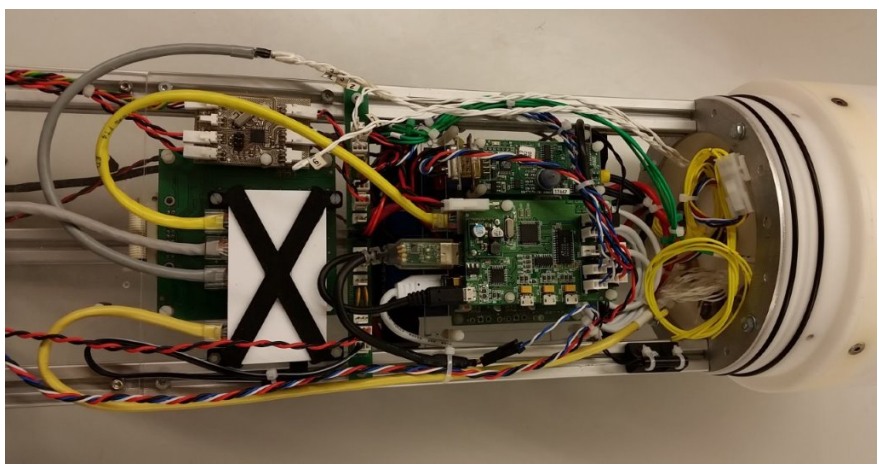

Fig. 3. Internal view of the pressure housing, with electronics and batteries.

\section{Additional Devices}

Taking advantage of the structural profiles and the real time link with the surface, an IP camera was mounted on the dock looking up, so that it is possible to visually monitor all docking operation. At the same time, another camera was installed looking down, to provide a simple means to assess the type of sea bottom during deployment and, afterward, to document any marine life in the vicinity of the docking station. Since the pressure vessel has many spare ports, it is straightforward to support additional subsystems, like water quality sensors.

\section{Surface Gateway}

For the real time supervision of the docking maneuver, there is a power and Ethernet cable linking the pressure housing of the docking station with the surface. At the surface, a WiFi link establishes the connection to a control interface (located on shore or on a mission support vessel).

The control interface allows the remote operation of the docking station, controlling its subsystems (fig. 4). More specifically, it can be used to switch on and off the electromagnets that hold the AUV in place, to control the intensity of the visual markers as well as the intensity of the lights associated to the video cameras attached to the docking station. The interface also displays information about the current status of the docking station, including real time images collected by the underwater cameras. When the AUV is docked, the interface also allows the download of data stored on the AUV computational system, as well as the upload of new configurations of missions to the AUV.

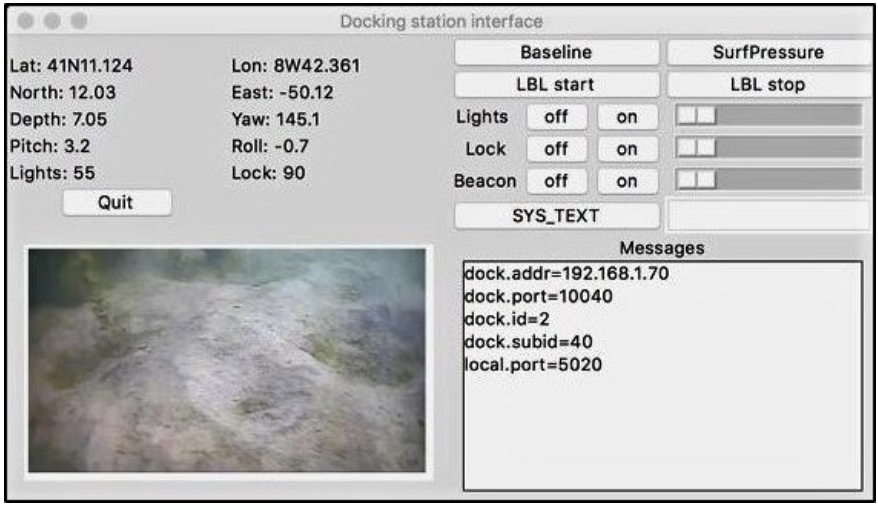

Fig. 4. Remote control interface.

\section{Operational Procedure}

The current configuration of the docking station is prepared for the two-stage navigation scheme described above. For the long range navigation, the AUV uses a long baseline acoustic positioning system based on two beacons installed in the operation area, such as described in [16] or in [17]. This ensures a global accuracy of a few meters, and a maximum range of more than $2 \mathrm{~km}$. The global position of the docking system is obtained in the same way, and it is provided to the AUV prior to the mission. When entering the docking maneuver, the AUV moves to the predefined position, with a given vertical offset so that the visual markers are visible, and then starts to look for the docking station. Upon positive detection of the visual markers, the AUV switches to a relative navigation scheme, based on visual information acquired by real time video processing, as already demonstrated in [10]. The AUV then descends towards the cradle on the docking station using visual servoing techniques. An electromagnet locking mechanism holds the vehicle in position, overcoming the small buoyancy of the vehicle, as well as any small disturbance forces. When standing at the dock, the AUV charges the batteries and communicates to shore using the docking station as the gateway.

\section{Customization of the MARES AUV}

One of the general guidelines for the development of the docking station was to have the least possible impact in the MARES AUV [1]. The docking maneuver uses video images from a small underwater camera, located at the bow and looking down, with LED lights to illuminate the target. In order to get into the vicinity of the docking station, the vehicle uses a standard LBL acoustic navigation system, together with data from the altimeter that provides height above the sea floor.

One of the required transformations of the MARES AUV was the addition of two disks of ferromagnetic material, so that two electromagnets located in the docking station can lock the vehicle in place and avoid unwanted relative motion. The main additional section was the wireless power transfer module, that connects to the internal battery management system to charge the batteries. 
A fifth thruster was also integrated in the MARES AUV to allow for independent sway motions. Although this additional degree of freedom is usually redundant in typical flight mode operations, it is essential to reject lateral disturbances in close range operations such as docking maneuvers with lateral currents.

MARES customization also included the integration of an auxiliary computer dedicated to image processing. This computer communicates with the main on-board computer through an Ethernet connection, and hosts an image processing system. This system, as described in [10], receives the images captured by the on board camera (as well as auxiliary navigation data - AUV global position, attitude, and height above bottom) in order to automatically detect the visual target and provide the position of the AUV with respect to the target, whenever it is present in the image.

Additionally, the MARES control software was also updated to include two additional maneuvers. The first look_for_dock () - is a searching maneuver, in which the AUV navigates at a constant altitude, sweeping, in parallel lines, an area around the estimated location of the docking station. The size of the area, the altitude of the AUV above ground, the spacing between lines, and the AUV velocity, depend on the accuracy of the acoustic positioning system and on the parameters of the underwater camera. This maneuver is completed as soon as the visual target is detected or the area is completely swept. The second maneuver final_approach ( ) - consists in the visual servoing of the vehicle towards the docking station until it reaches the cradle. It takes full advantage of the ability of the MARES AUV to move vertically in the water column at zero forward velocity and keeping a null pitch angle. During this maneuver, a control algorithm as described in [10] ensures that the horizontal position of the AUV converges to the horizontal position of the docking station, while the vehicle descends till the cradle.

In order to successfully dock the AUV, these two maneuvers are combined in a multiple retry loop that accommodates errors in the acoustic positioning system that might lead to uncovered areas during the look_for_dock() maneuver or disturbances in the AUV motion during the final_approach () maneuver that might lead to losing the visual target.

Figure 5 shows a perspective of the docking station, as seen from the AUV when it is trying to find it during the look_for_dock () searching phase. Note that the colored marker globes have a very dim internal light, but it is sufficient to highlight them in the dark background, and facilitate the identification with the AUV camera.

\section{EXPERIMENTAL VALIDATION}

The validation of the overall docking system followed a modular approach, where individual blocks were tested independently under relevant environmental scenarios. Concerning the MARES AUV, individual tests addressed the accuracy of the acoustic positioning system, the capability to detect targets

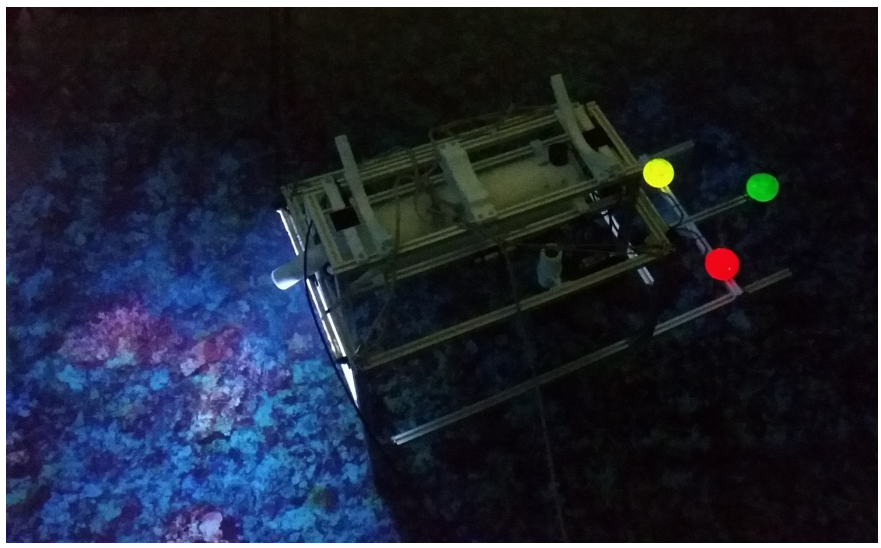

Fig. 5. The docking station, as seen from the AUV in the searching phase.

on the sea floor in area sweeping operations, and the ability to dock based on visual servoing techniques.

The acoustic positioning system used by the MARES AUV has been extensively tested in field trials and its accuracy fully characterized, as shown in [18]. Under static conditions, the positioning accuracy is better than 1 meter, for ranges to beacons up to 500 meters. This figure deteriorates with the increase of the vehicle velocity, reaching about 3 meters for MARES nominal velocity of $1 \mathrm{~m} / \mathrm{s}$ and typical geometric dilution of precision in the operation area.

The detection of visual targets lying on the sea floor was extensively tested during the euRathlon 2015 competition where the MARES AUV was part of a multi domain robotic team for search and rescue operations [19]. In these trials, MARES performed several sweeping maneuvers, similar to look_for_dock (), where it was able to detect and geolocate multiple visual targets distributed on the operation area. Geo-location accuracy ultimately depends on the acoustic positioning system accuracy and values similar to the above mentioned ones could be easily achieved.

The visual servoing system has already been tested in indoor environments (fig. 6). The accuracy of the visual positioning is 2 millimeters in the horizontal coordinates and 15 millimeters in the vertical coordinate [10]. These figures assure that the AUV can easily approach the docking station, aligning itself with the direction of the docking station and with the longitudinal position of the power transfer system and the electromagnetic holding devices. The visual servoing relies on a control algorithm that accounts for the nonlinearities of the system as described in [10].

\section{CONCLUSION AND Future WORK}

This paper described the development of a lightweight docking station for the MARES AUV, a modular hovering vehicle. The AUV approaches the docking station using acoustic navigation techniques and, when in close range, switches to a visual servoing mechanism, in which it visually tracks a set of visual markers for the final docking sequence. Once sitting in the docking cradle, a set of electromagnets holds the vehicle in position, while the batteries are charged and data is exchanged. 


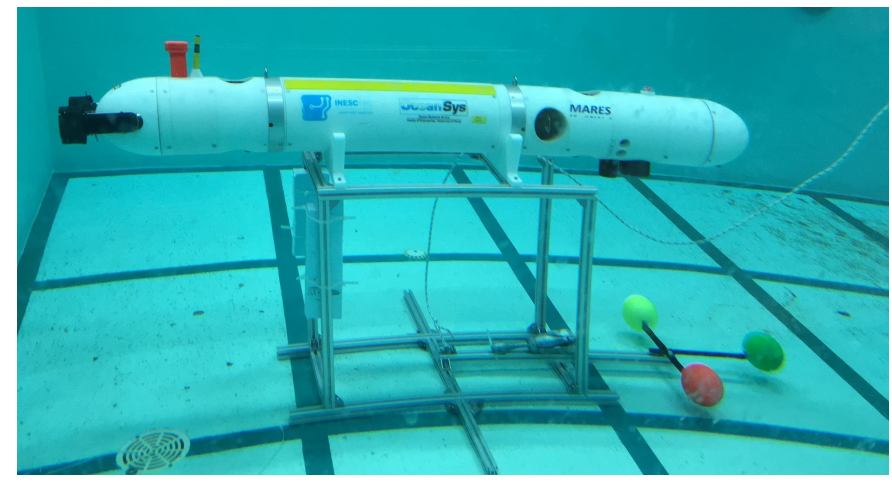

Fig. 6. The MARES AUV docking into a simplified docking station, using visual servoing.

The changes required for the MARES AUV to be able to dock were minor, taking advantage of the modular construction of the vehicle. The docking station was developed using a similarly modular approach, with a mix of off-the-shelf parts and the reuse of specific components from other projects. The current solution can be launched and recovered using a small vessel, and quickly reconfigured to match different vehicle versions. A similar approach can be used for other hovering type AUVs, paving the way to a common architecture for a generalized docking station.

The preliminary tests have demonstrated the effectiveness of our approach for the docking maneuver. These tests have been made in test tanks and controlled areas, and will be shortly followed by a series of demonstrations in field operations. The integration of navigation algorithms and motion primitives refined from other projects, maximizes the chances of immediate success, as well as the ability to adequately tackle any upcoming difficulties. Moreover, the docking station can easily be adapted to other configurations, like hanging from the surface. This will enlarge the range of application scenarios, although creating additional challenges, for example in terms of coordinated control of the docking station and the AUV.

The utilization of illuminated globes have provided excellent results in visual identification, under various lightning conditions. One additional feature that can easily be incorporated is the realtime measurement of ambient light, so that the docking station can adjust automatically the light intensity of the globes for dynamic lightning conditions.

\section{ACKNOWLEDGMENTS}

This article is a result of the project ENDURE (PT02_Aviso4_0015) supported by the EEA Grants Iceland, Liechtenstein and Norway.

This work is financed by the ERDF - European Regional Development Fund through the Operational Programme for Competitiveness and Internationalisation - COMPETE 2020 Programme within project "POCI-01-0145-FEDER-006961", and by National Funds through the Portuguese funding agency, FCT - Fundação para a Ciência e a Tecnologia as part of project "UID/EEA/50014/2013".
The research leading to these results has received funding from the European Union's Horizon 2020 - The EU Framework Programme for Research and Innovation $2014-2020$, under grant agreement No.692427.

\section{REFERENCES}

[1] N. A. Cruz and A. C. Matos, "The MARES AUV, a modular autonomous robot for environment sampling," in Proc. MTS/IEEE Int. Conf. Oceans 2008, Quebec, Canada, Sept. 2008.

[2] H. Singh, J. G. Bellingham, F. Hover, S. Lerner, B. A. Moran, K. V. der Heydt, and D. Yoerger, "Docking for an autonomous ocean sampling network," IEEE J. Oceanic. Eng., vol. 26, no. 4, pp. 498-514, Oct. 2001.

[3] R. Stokey, B. Allen, T. Austin, R. Goldborough, N. Forrester, M. Purcell, and C. V. Alt, "Enabling technologies for REMUS docking: An integral component of an autonomous ocean-sampling network," IEEE J. Oceanic. Eng., vol. 26, no. 4, pp. 487-497, Oct. 2001.

[4] B. Hobson, R. McEwen, J. Erickson, T. Hoover, L. McBride, F. Shane, and J. Bellingham, "The development and ocean testing of an AUV docking station for a 21" AUV," in Proc. MTS/IEEE Int. Conf. Oceans 2007, Vancouver, BC, Canada, Oct. 2007.

[5] Hydroid, a Kongsberg Company, "Underwater mobile docking of autonomous underwater vehicles," in Proc. MTS/IEEE Int Conf. Oceans 2012, Hampton Roads, VA, USA, Oct. 2012, pp. 1-15.

[6] B. M. Ferreira, A. C. Matos, N. A. Cruz, and A. P. Moreira, "Homing a robot with range-only measurements under unknown drifts," Robotics and Autonomous Systems, vol. 67, pp. 3 - 13, 2015, advances in Autonomous Underwater Robotics.

[7] P. Batista, C. Silvestre, and P. Oliveira, "A time differences of arrivalbased homing strategy for autonomous underwater vehicles," Int. J. Robust and Nonlinear Control, vol. 20, no. 15, pp. 1758-1773, 2010.

[8] J. Jouffroy and J. Reger, "An algebraic perspective to single-transponder underwater navigation," in IEEE Conf. on Computer Aided Control System Design, IEEE Int. Conf. on Control Applications, IEEE Int. Symp. on Intelligent Control, CACSD/CCA/ISIC, Munich, Germany, Oct. 2006, pp. 1789-1794.

[9] A. Figueiredo, B. Ferreira, and A. Matos, "Tracking of an underwater visual target with an autonomous surface vehicle," in Proc. MTS/IEEE Int. Conf. Oceans 2014, St John's, NL, Canada, Sept 2014, pp. 1-5.

[10] - "Vision-based localization and positioning of an AUV," in Proc. MTS/IEEE Int. Conf. Oceans 2016, Shanghai, China, April 2016, pp. $1-6$.

[11] H. Kondo, K. Okayama, J. K. Choi, T. Hotta, M. Kondo, T. Okazaki, H. Singh, Z. Chao, K. Nitadori, M. Igarashi, and T. Fukuchi, "Passive acoustic and optical guidance for underwater vehicles," in Proc. MTS/IEEE Int. Conf. Oceans 2012, Yeosu, Korea, May 2012, pp. 1-6.

[12] M. Wirtz, M. Hildebrandt, and C. Gaudig, "Design and test of a robust docking system for hovering AUVs," in Proc. MTS/IEEE Int. Conf. Oceans 2012. Hampton Roads, VA, USA: Oceans 2012, Oct. 2012.

[13] T. Maki, R. Shiroku, Y. Sato, T. Matsuda, T. Sakamaki, and T. Ura, "Docking method for hovering type AUVs by acoustic and visual positioning," in Proc. Int. Symp. Underwater Tech. UT'13, Tokyo, Japan, 2013.

[14] H. Yoshida, S. Ishibashi, O. Yutaka, M. Sugesawa, and K. Tanaka, "A concept design of underwater docking robot and development of its fundamental technologies," in Proc. IEEE/OES Int. Conf. AUV 2016, Tokyo, Japan, Nov 2016, pp. 408-411.

[15] N. A. Cruz, A. C. Matos, and B. M. Ferreira, "Modular building blocks for the development of AUVs - from MARES to TriMARES," in Proc. Int. Symp. Underwater Tech. UT'13, Tokyo, Japan, Mar. 2013.

[16] R. Almeida, N. Cruz, and A. Matos, "Synchronized intelligent buoy network for underwater positioning," in Proc. IEEE Int. Conf. Oceans'10, Sydney, Australia, May 2010.

[17] —, "Man portable acoustic navigation buoys," in Proc. MTS/IEEE Int. Conf. Oceans 2016, Shanghai, China, April 2016, pp. 1-6.

[18] R. Almeida, J. Melo, and N. Cruz, "Characterization of measurement errors in a LBL positioning system," in Proc. MTS/IEEE Int. Conf. Oceans 2016, Shanghai, China, April 2016, pp. 1-6.

[19] A. Matos, A. Martins, A. Dias, B. Ferreira, J. Almeida, H. Ferreira, G. Amaral, A. Figueiredo, R. Almeida, and F. Silva, "Multiple robot operations for maritime search and rescue in euRathlon 2015 competition," in Proc. MTS/IEEE Int. Conf. Oceans 2016, Shanghai, China, April 2016, pp. 1-6. 